\title{
Genome Profiling for Health Promoting and Disease Preventing Traits Unraveled Probiotic Potential of Bacillus clausii B106
}

\author{
N.G. Kapse' ${ }^{1}$ A. S. Engineer ${ }^{1}$, V. Gowdaman' ${ }^{1}$ S. Wagh ${ }^{2}$, and P. K. Dhakephalkar ${ }^{1 *}$ \\ ${ }^{1}$ Bioenergy Group, MACS-Agharkar Research Institute, G.G. Agarkar Road, Pune-411004, India \\ ${ }^{2}$ Hi Tech BioSciences India Ltd., Research \& Development Centre, Plot No. 6\& 8, Ambadvet Industrial Estate, PO Paud, Pune-412108, India
}

Received: April 5, 2018 / Revised: August 30, 2018 / Accepted: September 19, 2018

\begin{abstract}
Spore-forming Bacillus species are commercially available probiotic formulations for application in humans. They have health benefits and help prevent disease in hosts by combating entero-pathogens and ameliorating antibiotic-associated diarrhea. However, the molecular and cellular mechanisms of these benefits remain unclear. Here, we report the draft genome of a potential probiotic strain of Bacillus clausii B106. We mapped and compared the probiotic profile of B106 with other reference genomes. The draft genome analysis of B106 revealed the presence of ADI pathway genes, indicating its ability to tolerate acidic pH and bile salts. Genes encoding fibronectin binding proteins, enolase, as well as a gene cluster involved in the biosynthesis of exopolysaccharides underscored the potential of B106 to adhere to the intestinal epithelium and colonize the human gut. Genes encoding bacteriocins were also detected, indicating the antimicrobial ability of this isolate. The presence of genes encoding vitamins, including Riboflavin, Folate, and Biotin, also indicated the health-promoting ability of B106. Resistance of B106 to multiple antibiotics was evident from the presence of genes encoding resistance to chloramphenicol, $\beta$-lactams, Vancomycin, Tetracycline, fluoroquinolones, and aminoglycosides. The findings indicate the significance of $B$. clausii B106 administration during antibiotic treatment and its potential value as a probiotic strain to replenish the health-promoting and disease-preventing gut flora following antibiotic treatment.
\end{abstract}

Keywords: Probiotics, Bacillus, spore formers, genome analysis

\section{Introduction}

Probiotics are live microbial supplements that aid in improving the intestinal microbial balance and impart health benefit on the host [1]. Lactobacillus are the most commonly used probiotic bacteria, however their shelflife is very small. From last five decades, spore forming Bacillus have been marketed as probiotic preparations. The advantage of endospore forming Bacillus species over non-spore formers like Lactobacillus is their long

\section{*Corresponding author}

Tel: +91 020 25325096, Fax: +912025651542

E-mail: pkdhakephalkar@aripune.org

(c) 2018, The Korean Society for Microbiology and Biotechnology shelf life and high viability under hostile conditions [2]. The Bacillus spores help them to survive the harsh conditions during gastrointestinal transit (GIT), which has been established by a study in murine model wherein the consumed Bacillus spores could survive, propagate, flourish and also re-sporulate on reaching the gut [3].

Several studies have proved the acid-bile tolerance, mucin/fibronectin binding, anti-oxidant, antimicrobial and immuno-modulatory ability and safety of probiotic Bacillus strains [4-7]. Bacillus spp. also provide essential nutrient supplements to the gut microflora and the host by improving nutritional amenability of complex food and producing micronutrients like essential amino acids and vitamins [8]. 
Bacillus probiotics are currently gaining relevance in probiotic industry because of their spore-forming attribute with indefinite shelf life. Strains of B. subtilis, $B$. licheniformis, B. cereus, B. pumilus, B. clausii and B. coagulans, other spore-formers like Paenibacillus polymyxa and Brevibacillus laterosporus are currently marketed as spore forming probiotics [9]. Each strain possess unique probiotic properties and thus, every potential probiotic strain needs to be characterized for their specific probiotic characteristics [10].

Bacillus spp. are well known for their disease preventing properties, acting as antidiarrheal agent to bacterial dysentery \& antibiotic-associated diarrhea and thus, inhibiting enteric pathogens [7, 8]. B. clausii is a prolific source of large numbers of bacteriocins, lytic enzymes and antibiotics with antibacterial activity. For example, clausin, a known lantibiotic active against Clostridium difficile and Staphylococcus aureus can be used as a potential alternative to the currently used antibiotics in future [11]. The Bacillus probiotic Enterogermina containing 4 strains of Bacillus clausii (O/C, N/R, SIN, and $\mathrm{T})$ is well documented for beneficial clinical effects, especially in the treatment of diarrhea and in the prevention of infectious diseases. However, the mechanistic insights at gene level for such beneficial probiotic attributes remains largely unexplored [11]. Genome scale analysis of only two strains of B. clausii (UBBC07 and AKU0647) are documented, highlighting the paucity of information available on probiotic characterization of $B$. clausii at gene level.

Hence, the draft genome analysis of Bacillus clausii B106 was carried out to explore its probiotic potential. The genome of our strain shared 100\% similarity with genomes of strains KSM-K16, ENTpro and UBBC07. Experimental data suggest UBBC07 to be safe for human consumption as probiotic it is non-hemolytic and does not possess toxin genes and transferable antibiotic resistance genes [10]. Furthermore, in vitro analyses revealed that $\mathrm{UBBC} 07$ can tolerate and survive harsh gastric conditions and adhere to intestinal cells [12]. The genome analysis revealed the presence of genes associated with adhesion, antibiotic resistance- and stress resistance-related domains, which may help us in unraveling the mechanisms of survival in harsh conditions of gastric transit and adhesion to intestinal cells. Sequencing and annotation of Bacillus clausii B106 genome gave insights into the molecular basis of probiosis in B106.

\section{Materials and Methods}

\section{Bacterial strain, DNA preparation and Genome Sequencing}

Bacillus clausii B106 was isolated from healthy human and cultured in Nutrient broth at $37^{\circ} \mathrm{C}$. Genomic DNA from the strain B106 was isolated using GenElute $^{\mathrm{TM}}$ Bacterial Genomic DNA Isolation kit (Sigma, USA) using manufacturers protocol. The genome of B106 was sequenced by shotgun sequencing method performed using the Ion Torrent PGM platform on a $318^{\mathrm{TM}}$ chip with 400-bp chemistry (Life Technologies, USA).

\section{Genome assembly and annotation}

De novo genome assembly was carried out using SPAdes assembler version 3.9.1 [13]. Functional annotation of the B106 genome was carried out by RAST server (Rapid Annotation using Subsystem Technology) [14]. The genome was mined for the presence of probiotic marker genes associated with health promotion and disease prevention by using RAST server. The individual probiotic attributes are described alongwith their Figfam numbers. FIGfam is a set of proteins that are believed to be isofunctional homologs, having the same function and are derived from common ancestor. Any user can go to the RAST server (http://rast.nmpdr.org/) and login using user id as "guest" and password as "guest" and access the complete annotation details alongwith the figfam numbers for the described $B$. clausii strain B106 with ID 66692.20.

The genome sequence of Bacillus clausii B106 has been deposited in GenBank under the accession number NFZO00000000.

\section{Comparative genome analysis}

Genomes of four B. clausii strains namely (KSMK16, ENTpro, AKU0647 and UBBC07) were obtained from NCBI database for comparative analysis with B106. For in-silico genome to genome comparison, Digital DNADNA hybridization was carried out using B106 as reference, as described by Auch et al. (2010) using online tool http://ggdc.dsmz.de/) [15]. Blast Ring Image Generator (BRIG) software was used to generate comparative circular genome image. The genome sequences of query and reference were submitted in the 'fasta' format to 
generate the image with $B$. clausii B106 as reference strain. Nucleotide alignments of multiple query genomes against the reference strain were generated using BLASTn to create the circular genome comparison map [16].

\section{Prediction of secondary metabolite and bacteriocin pro- duction}

antiSMASH web server was used to predict the antibiotic and secondary metabolite biosynthetic gene clusters from the genome of B106. antiSMASH uses a large number of in silico secondary metabolite analysis tools, integrates them and provides the prediction [17]. The bacteriocin mining was executed in BAGEL 3 web based server using the 'fna' file from B. clausii B106. The software works with input data evaluated against a curated dataset of bacteriocins [18].

\section{Antibiotic resistance prediction}

Antibiotic resistance genes in the genome of B106 was predicted using the web based server CARD, a thoroughly curated collection of identified resistance genes and related antibiotics by the Antibiotic Resistance Ontology (ARO) and AMR gene detection models. CARD analyses the genome sequences, using BLAST and the Resistance Gene Identifier (RGI) software for prediction [19].

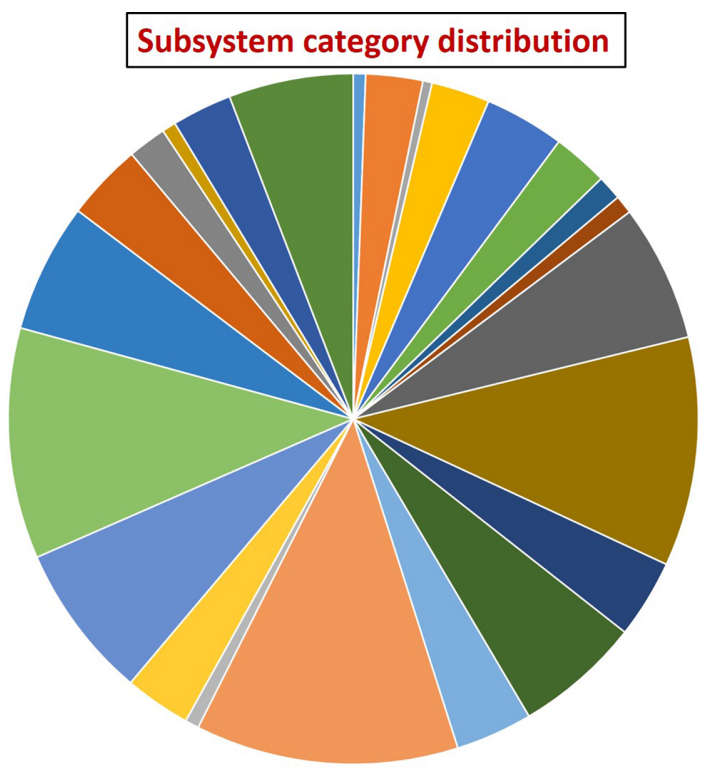

\section{Results and Discussion}

\section{Taxonomic affiliation of the strain B106}

Identification of a strain offers a myriad of information such as its typical habitat, origin, strain differentiation and may also indicate the strain's safety and its applicability for use in commercial products [20]. The strain B106 was identified as Bacillus clausii based on its 16S rRNA gene sequencing [21] by BLASTn similarity search, it showed 99\% similarity with Bacillus clausii DSM 8716.

Based on the taxonomic characterization, the strain B106 was designates to be a different strain of B. clausii. The probiotic potential varies from strain to strain hence the whole genome analysis of B106 was carried out to study its probiotic potential.

\section{General genome architecture}

Bacillus clausii B106 genome was assembled into 45 contigs forming one presumptive circular chromosome of 4253129 base pairs with $44.6 \%$ GC content. A total of 477 subsystems (Fig. 1, Table S1), 4710 protein-coding genes, and 80 RNA genes were predicted using RAST. $49 \%$ of the protein-coding genes were specified as a putative function.

\section{In silico Digital DNA-DNA Hybridization}

With the genome of B106 available, in silico DNA-

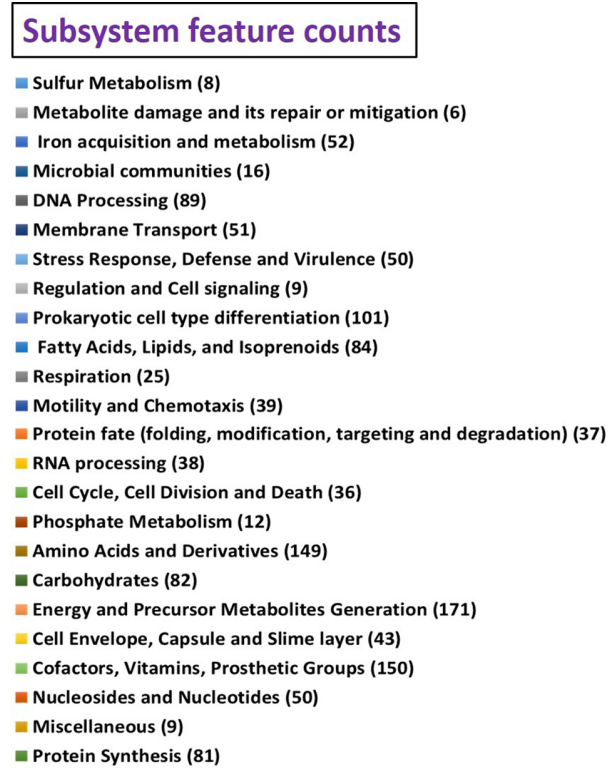

Fig. 1. Genome annotation of B106 by Rapid Annotation using Subsystem Technology (RAST). 
Table 1. Comparative genome analysis of B106 with other publically available strains of Bacillus clausii (PGAAP data).

\begin{tabular}{ccccccc}
\hline Strain & Size (Mb) & \%DNA-DNA Hybridization & GC\% & Genes & rRNA & tRNA \\
\hline B106 & 4.25 & 100 & 44.6 & 4326 & 14 & 69 \\
KSM-K16 & 4.30 & 100 & 44.8 & 4329 & 22 & 75 \\
ENTPro & 4.29 & 100 & 44.7 & 4245 & 22 & 75 \\
UBBC-07 & 4.19 & 100 & 44.6 & 4178 & 9 & 75 \\
AKU0647 & 4.65 & 50.60 & 44.3 & 4611 & 13 & 75 \\
\hline
\end{tabular}

DNA-hybridization (DDH) values were calculated for publically available genome of B. clausii strains (KSMK16, ENTpro, AKU0647 and UBBC07) using GenomeGenome Distance Calculator (GGDC) 2.0 server [15]. Our strain B106 shared 100\% DDH value with 3 strains KSM-K16, ENTpro and UBBC07 (Table 1).

\section{BRIG analysis for comparative genome analysis}

The genomic attributes of the genomes used for comparison are summarized in Table 1. Publically available B. clausii genomes were used for the analysis. B. clausii strain KSM-K16 has the highest $\mathrm{G}+\mathrm{C}$ content, $44.8 \%$, whereas the lowest $\mathrm{G}+\mathrm{C}$ contents of $44.3 \%$, was from $B$. clausii strain AKU0647. The genome sizes of the B. clausii strains range from $\sim 4.19 \mathrm{Mb}$ to $\sim 4.65 \mathrm{Mb}$. The circular image comparing the B. clausii genomes with B106 is given in Fig. 2.

The gap in the circular image between the reference B106 and the query genome AKU0647 indicates the dif-

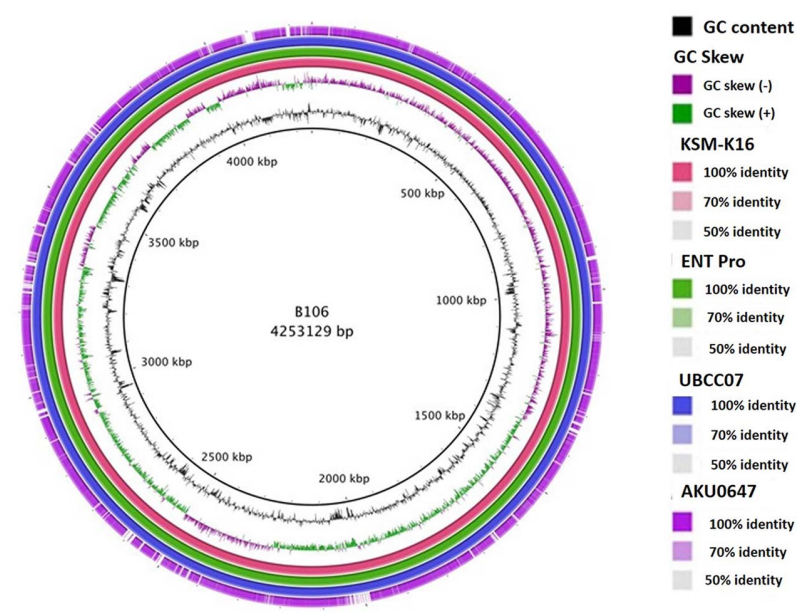

Fig. 2. Circular comparison of Bacillus clausii B106 as a reference. Each colored ring represents a query genome. The intensity of the color represents relative levels of nucleotide homology between the reference and query genomes. ferences between the strains of B. clausii with B106, indicating B106 to be a different strain from AKU0647. Our strain B106 was found to be $100 \%$ similar to ENTPro, UBBC-07 and KSM-K16 as evident from complete circles in the BRIG image, further supporting DDH results. Also, it proved the completeness of our strain in terms of sequencing.

\section{Annotation of the genomes}

Gene prediction and functional annotation carried out by RAST, revealed the presence of genes associated with survival under harsh conditions in the Gastro Intestinal Tract (GIT), bacterial adhesion and aggregation along with the genes required for vitamin synthesis in B106. Also, several genes involved in stress resistance including two toxin-antitoxin gene pairs were detected in the genome.

Genome mining revealed genes encoding for industrially important enzymes like Alpha-glucosidase (EC 3.2.1.20), Alpha-N-arabinofuranosidase (EC 3.2.1.55), Arabinogalactan endo-1,4-beta-galactosidase (EC 3.2.1.89), Beta-galactosidase (EC 3.2.1.23), Alpha-galactosidase (EC 3.2.1.22), Endoglucanase (EC 3.2.1.4), lipase (esterase), Pectate lyase precursor (EC 4.2.2.2), Protease and Extracellular serine protease in B106 genome, highlighting the biotechnological potential of the strain.

Adaptation to acidic conditions and low pH: On passing through the oral route, the first encounter that the bacterium faces is the hostile acidic environment of the gastro-intestinal tract (GIT). For adaptation of the organism in such hostile GIT, Sigma B (an alternative sigma factor) plays a significant role [22, 23]. Another approach by which the organism survives the low $\mathrm{pH}$ in the GIT is by $\mathrm{pH}$ homeostasis contributed by Arginine Deiminase (ADI) pathway [24]. In a characteristic bacterial ADI pathway, extracellular arginine is fed into the 
ADI system through arginine/ornithine antiporter [25]. It is to be also noted that the ADI pathway is also controlled by Sigma B factor [26]. B106 genome encodes Sigma B factor, arginine/ornithine antiporter ArcD, Ornithine carbamoyl-transferase and Arginine pathway regulatory protein ArgR, repressor of arg regulon, indicating the strain's ability to adapt to extreme $\mathrm{pH}$ conditions and Bile salt in GIT.

To survive under acidic conditions of the stomach, the probiotic bacteria require genes encoding for symporter that can help them thrive within the GIT for considerable amount of time [27]. B106 possesses genes encoding for $\mathrm{Na}^{+} / \mathrm{H}^{+}$(4 copies), sugar $/ \mathrm{H}^{+}$and glutamate $/ \mathrm{H}^{+}$symporters that are essential for survival under acidic conditions. RAST annotation also revealed the presence of Bile acid - sodium symporter which is known to provide resistance to bile salt in bacteria [28].

Genes involved in bile salt tolerance like Bile salt hydrolase (BSH) and choloylglycine hydrolase were absent in B106. Probiotic organisms isolated from the GIT often exhibit BSH activity (L. gasseri, L. johnsonii, L. acidophilus, some strains of L. plantarum and Bifidobacterium species), whereas bacteria isolated from other sources like fermented products and vegetables do not exhibit BSH activity [29], as they do not contain bile acids. Inactivation of genes coding for BSH enzymes such as bshA and bshB, did not affect the bile tolerance in L. acidophilus [30]. Another report established that absence of BSH does not affect GIT perseverance capacity of a strain, as seen in L. johnsonii NCC533, a tripleknockout mutant of BSH proteins [31]. Absence of BSH does not make B106 sensitive to gastric transit, as it also possess $\mathrm{ADI}$ pathway for bile tolerance.

Colonization of intestinal epithelium: One of the most important features for a probiotic strain is adhesion to intestinal epithelium [32]. Probiotics also positively affect the host defense mechanism by reducing the pathogenic adherence through mucin and fibrinogen binding proteins therefore creating an important host defense mechanism against infection [33]. Genome annotation of B106 revealed various marker genes exerting probiotic mechanism associated with adhesion and aggregation that is summarized in Table 2.

Enolase is a binding protein produced by probiotic bacteria that aids in adherence in GIT [34]. Genome analysis
Table 2. Putative genes involved in adhesion and aggregation in B106.

\begin{tabular}{lc}
\hline \multicolumn{1}{c}{ Putative genes } & Figfam \\
\hline Enolase & FIG00000118 \\
Sortase linked LPTXG motifs & FIG00007328 \\
Fibronectin binding protein & FIG00138381 \\
EPS biosynthetic gene clusters & FIG00009775 \\
a. Undecaprenyl-phosphate galactosephospho- & FIG00002968 \\
transferase & FIG00002620 \\
b. Eps C & FIG00035701 \\
c. Eps D & FIG00002449 \\
d. Manganese-dependent protein-tyrosine phos- \\
$\quad$ phatase & \\
Triosephosphate isomerase & FIG00000076 \\
Flagellar hook-associated protein Fli D & FIG00000668 \\
Flagellar hook-associated protein Flg L & FIG00009398 \\
Flagellar hook-associated protein Flg K & FIG00000650 \\
\hline
\end{tabular}

of B106 revealed the presence of gene encoding enolase. Mucin binding protein was not detected in B106, but there was a single copy of fibronectin-binding protein that aids in adhesion of the bacterium to host fibronectin. Three proteins with the LPxTG-type anchor were identified in the B106 genome, which is involved in cell surface localization and interaction with peptidoglycan.

Exopolysaccharide (EPS) biosynthetic gene clusters were detected in B106 genome. EPS biosynthesis by probiotic strains is thought to be associated with adherence to intestinal mucus and also antagonize the pathogenic activity of enteropathogens [35]. The probiotic bacteria also release EPS into the surrounding environment, to protect themselves during starvation and also from extreme $\mathrm{pH}$ and temperature [36]. Apart from these factors, flagellum can directly act as an adhesin and play an essential role in colonization by facilitating bacterial motility [37]. There were four flagellar hook associated proteins (Table 2) identified in B106, which may be responsible for adhesion and colonization.

Interaction with other microorganisms: Inhibition of entero-pathogens through production of bacteriocins: Previous studies have reported that antimicrobial compounds such as bacteriocin, colicin, etc. are secreted by probiotic bacteria, conferring disease preventing properties to fight against intestinal pathogenic bacteria like Helicobacter pylori, Gardenerella vaginalis, Listeria 

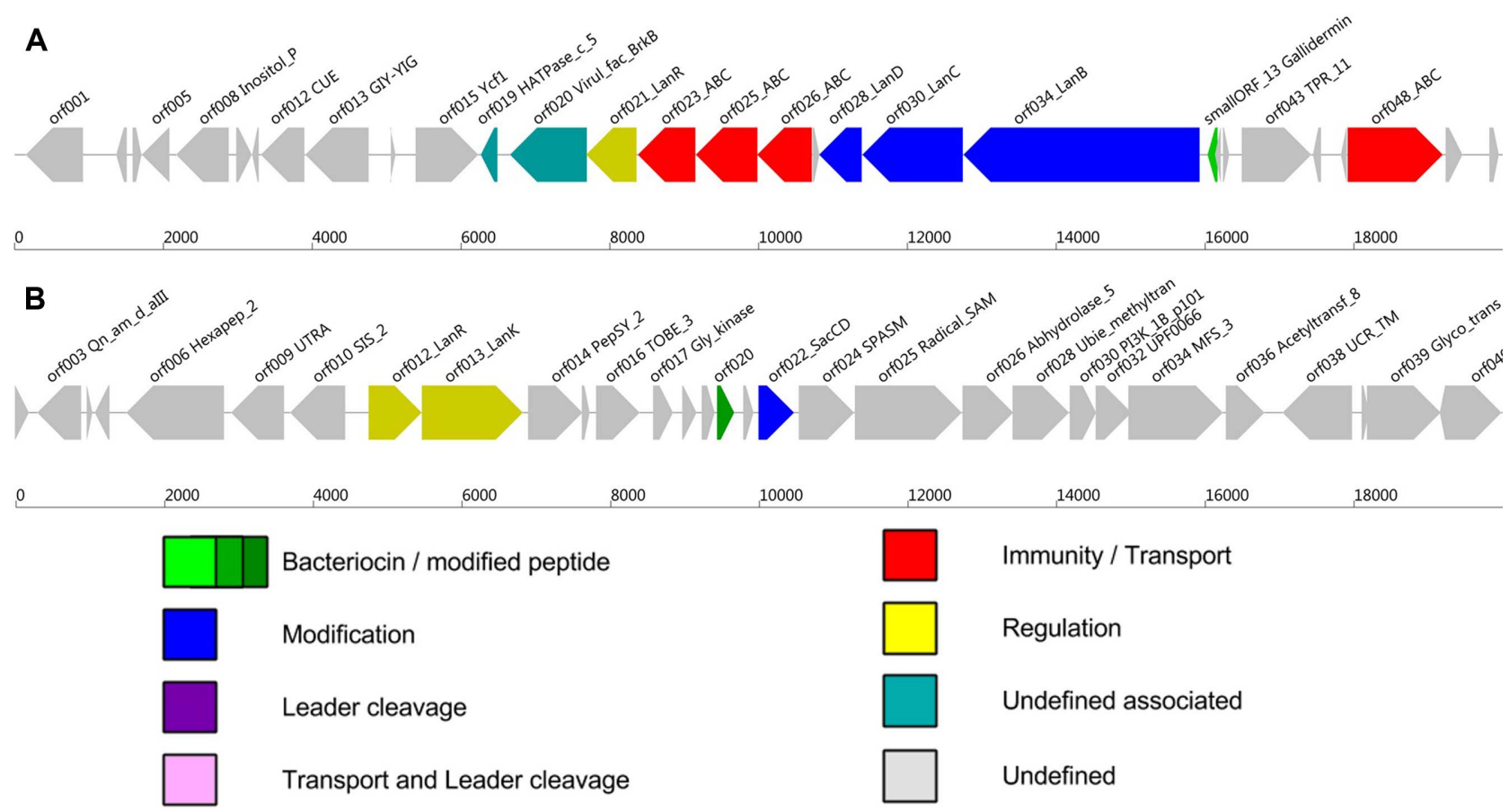

Fig. 3. (A) Lantipeptide/Class I bacteriocin predicted on orf_13 and (B) Sactipeptide bacteriocin predicted on orf020 in B106 using BAGEL.

monocytogenes, Clostridium difficile, Staphylococcus pneumonia and Streptococcus aureus [38]. The genome of B106 was also annotated with genes encoding antibacterial peptides like Bacteriocins (Bacitracin) and Colicin (colicin V) that kills sensitive cells by disrupting their membrane potentials, suggesting B106 can achieve "competitive exclusion" by secreting antimicrobial substances [39].

Putative bacteriocins were predicted by using the BAGEL 3. Bacteriocins are classified into three classes by BAGEL: (i) lanthionine-containing bacteriocins/lantibiotics, (ii) non-lanthionine-containing bacteriocins and (iii) bacteriolysins/non- bacteriocin lytic proteins [40].

In B106, BAGEL predicted two bacteriocins, class I Lantipeptide and other from class Sactipeptide (Fig. 3). The class I lantipeptide was identified as Gallidermin/
Nisin, a polycyclic antibacterial peptide commonly produced by the bacterium Lactococcus lactis [41]. Nisin is a broad-spectrum bacteriocin, active against Gram-positive bacteria, such as Staphylococcus aureus, Listeria monocytogenes, Clostridium botulinum, etc. and also effective against spores [41]. The protein sequence of the other bacteriocin predicted from class Sactipeptide showed similarity to a hypothetical protein (Table 3).

Another mechanism, by which the probiotic bacteria exhibits antimicrobial activity, is by $\mathrm{H}_{2} \mathrm{O}_{2}$ production [42], as it has been proposed to be one of the important antimicrobial property. B106 strain did not possess pyruvate oxidase, $\mathrm{NADH}$ oxidase or Lactate oxidase; the genes commonly involved in $\mathrm{H}_{2} \mathrm{O}_{2}$ production. However, there were several other genes responsible for hydrogen peroxide production, which are reported to be linked

Table 3. Identified putative bacteriocin(s) or modified peptide(s).

\begin{tabular}{ccll}
\hline Type & Protein ID & \multicolumn{1}{c}{ Protein sequence } & \multicolumn{1}{c}{ Identity } \\
\hline Lantipeptide Class I & AOI_1;small ORF_13 & MEKAFDLDLEVVHTKAKDVQPDFTSVSFCTPGCGETGS- & $\begin{array}{l}\text { Gallidermin/nisin family lantibiotic } \\
\text { (Bacillus) }\end{array}$ \\
& & FNSFCC & VLLFMTMVALNDCLTFPISSKALLIYFEEKPSFKANDLFLFE \\
Sactipeptides & AOI_1;orf020 & Hypothetical protein DB29_01543 \\
& & LLTGIGLFVNHHIFQYVLVMALLVNLSKMRVSA & (Bacillus clausii) \\
\hline
\end{tabular}


Table 4. Putative genes involved in $\mathrm{H}_{2} \mathrm{O}_{2}$ production in B106.

\begin{tabular}{lc}
\hline \multicolumn{1}{c}{ Putative genes } & Figfam \\
\hline $\begin{array}{l}\text { Cytochrome d ubiquinol oxidase subunit I } \\
\text { (EC 1.10.3.-) }\end{array}$ & FIG00096593 \\
$\begin{array}{l}\text { Cytochrome d ubiquinol oxidase subunit II } \\
\text { (EC 1.10.3.-) }\end{array}$ & FIG00000390 \\
$\begin{array}{l}\text { Glutathione peroxidase family protein } \\
\text { Pyridoxamine 5'-phosphate oxidase (EC 1.4.3.5) }\end{array}$ & FIG00133002 \\
Rrf2-linked NADH-flavin reductase & FIG01308892 \\
\hline
\end{tabular}

with $\mathrm{H}_{2} \mathrm{O}_{2}$ production especially NADH flavin reductase (Table 4). Even though the other essential oxidase genes were absent, presence of NADH flavin reductase [42], makes B106 a potential probiotic candidate with disease prevention trait.

Presence of Sporulation genes: Spore forming probiotic bacteria have an implausible benefit over non-spore formers as spores are highly resistant to extreme environmental settings [43]. In the genome of $B$. clausii B106 around 85 essential sporulation genes have been identified, suggesting the ability of spore forming B106 to survive acidic $\mathrm{pH}$ in the GIT and reach the small intestine unharmed. This further proved the robustness of B106.

Interaction with food components: presence of enzymes improving nutritional amenability of food: There are different kinds of food such as those that provide basic nutritional requirements for growth and other food components that exert beneficial effects e.g. functional food and nutraceuticals [44]. The metabolic activity of the gut microbiota on these food components can modify the host exposure to these components and also their probable health effects [45, 46]. Therefore, B106 genome was mined for genes involved in metabolism of complex carbohydrates, proteins and fats. Beta-glucosidase was the most abundant enzyme detected in the strain B106, involved in utilization of complex dietary polysaccharides and contributing to energy yield. Certain glycosidases like arabinofuranosidases and xylosidases, are required for complete degradation of complex polysaccharides such as arabinans and arabinoxylans [47, 48], were also encoded by B106, and in additional it is also equipped with genes and enzymes (two alpha-galactosi- dase and three beta galactosidase) for improving lactose intolerance [49].

Carbohydrate active enzyme were predicted which showed 165 genes encoding for carbohydrate active enzymes including 31 glycosyl transferase, 21 carbohydrate binding module, 66 glycosidase hydrolase, 5 polysaccharide lyase family, 35 carbohydrate esterase and 7 auxiliary activity. The presence of different subfamilies of glycosidase hydrolase and carbohydrate esterase suggests that the strain B106 can ferment various complex polysaccharides, an important competitive feature among bacteria in the large intestine [50]. Presence of proteases and lipases (esterases) in B106 further highlighted the ability of the strain to breakdown complex dietary components and make the food nutritionally amenable to the host.

Genetic determinants associated with probiotic characters exhibiting health promotion: The nutrient synthesis by probiotics in the GIT may enhance the nutrition availability by supplementing essential dietary nutrients for the host and the microbiome [51]. The genome of B. clausii B106 comprises of genes required for synthesis of B-vitamins like B2 (Riboflavin), B7 (Biotin) and B9 (Folate), a necessary feature of probiotics that provides essential vitamins to the host [27]. Probiotics that produce essential nutrients and vitamins maybe considered as nutritive supplements for vitamin or amino acid deficient individuals [52]. Genes detected in B106 that are involved in the vitamin biosynthesis are illustrated in Table 5.

Stress responsive proteins: A number of genes are involved in stress related responses such as heat shock proteins (HSPs) and Chaperons DnaJ and DnaK (detected in B106 genome), that imparts resistance to heat shock and other oxidative stress [28]. These HSPs and chaperons are necessary for normal cellular functions like growth, stability of nucleic acids and also known to inhibit the formation of inclusion bodies [53]. Oxidative stress is caused due to increased activity of reactive oxygen species (ROS) through the oxidation process. Probiotic bacteria are well known for their radical scavenging ability, and B106 genome harbored genes involved in the elimination of reactive oxygen species, such as genes encoding thioredoxin system (8 genes), 
Table 5. Putative vitamin biosynthetic proteins/genes detected in B106.

\begin{tabular}{|c|c|c|}
\hline Vitamin & Figfam & Putative biosynthesis Protein/gene/system detected in the B106 \\
\hline \multirow[t]{11}{*}{ Riboflavin } & FIG00000384 & $\begin{array}{l}\text { a. Diaminohydroxyphosphoribosylaminopyrimidine deaminase (EC 3.5.4.26) / 5-amino-6-(5-phos- } \\
\text { phoribosylamino)uracil reductase (EC 1.1.1.193) }\end{array}$ \\
\hline & & b. Molybdopterin binding motif, CinA N-terminal domain / C-terminal domain of CinA type S \\
\hline & FIG00000047 & c. Ribulose-phosphate 3-epimerase (EC 5.1.3.1) \\
\hline & FIG00000273 & d. Riboflavin synthase eubacterial (EC 2.5.1.9) \\
\hline & FIG00000378 & e. 3,4-dihydroxy-2-butanone 4-phosphate synthase (EC 4.1.99.12) / GTP cyclohydrolase II (EC 3.5.4.25) \\
\hline & FIG00000231 & f. 6,7-dimethyl-8-ribityllumazine synthase (EC 2.5.1.78) \\
\hline & FIG00056212 & g. Pyridine nucleotide-disulfide oxidoreductase; NADH dehydrogenase (EC 1.6.99.3) \\
\hline & & h. Diacylglycerol kinase (EC 2.7.1.107) \\
\hline & FIG00000862 & $\begin{array}{l}\text { i. Phosphoribosyl-AMP cyclohydrolase (EC 3.5.4.19) / Phosphoribosyl-j. ATP pyrophosphatase (EC } \\
\text { 3.6.1.31) }\end{array}$ \\
\hline & FIG00000811 & j. ATP phosphoribosyltransferase (EC 2.4.2.17) \\
\hline & FIG00008708 & k. NADH dehydrogenase (EC 1.6.99.3) \\
\hline \multirow[t]{7}{*}{ Biotin } & & a. Biotin operon repressor / Biotin-protein ligase (EC 6.3.4.15) \\
\hline & FIG00079247 & b. predicted biotin regulatory protein BioR (GntR family) \\
\hline & FIG01322255 & c. Acetoacetyl-CoA synthetase (EC 6.2.1.16) / Long-chain-fatty-acid--CoA ligase (EC 6.2.1.3) \\
\hline & FIG00012547 & d. Adenosylmethionine-8-amino-7-oxononanoate aminotransferase (EC 2.6.1.62) \\
\hline & & $\begin{array}{l}\text { e. Competence protein } \mathrm{F} \text { homolog, phosphoribosyltransferase domain; protein } \mathrm{YhgH} \text { required for uti- } \\
\text { lization of DNA as sole source of carbon and energy }\end{array}$ \\
\hline & FIG00012067 & f. Substrate-specific component BioY of biotin ECF transporter \\
\hline & FIG00033705 & g. Biotin synthesis protein BioC \\
\hline \multirow[t]{13}{*}{ Folate } & FIG00000048 & a. Dihydrofolate reductase (EC 1.5.1.3) \\
\hline & FIG00000252 & b. 5-formyltetrahydrofolate cyclo-ligase (EC 6.3.3.2) \\
\hline & FIG00000106 & c. Dihydrofolate synthase (EC 6.3.2.12) @ Folylpolyglutamate synthase (EC 6.3.2.17) \\
\hline & FIG00000440 & d. Pantoate--beta-alanine ligase (EC 6.3.2.1) \\
\hline & FIG00008304 & e. Aspartate 1-decarboxylase (EC 4.1.1.11) \\
\hline & FIG00000226 & f. GTP cyclohydrolase I (EC 3.5.4.16) type 1 \\
\hline & FIG00000171 & g. 2-amino-4-hydroxy-6-hydroxymethyldihydropteridine pyrophosphokinase (EC 2.7.6.3) \\
\hline & FIG00000373 & h. Dihydroneopterin aldolase (EC 4.1.2.25) \\
\hline & FIG00000307 & i. Dihydropteroate synthase (EC 2.5.1.15) \\
\hline & FIG00000708 & j. Aminodeoxychorismate lyase (EC 4.1.3.38) \\
\hline & FIG00341164 & k. Para-aminobenzoate synthase, aminase component (EC 2.6.1.85) \\
\hline & FIG01305088 & $\begin{array}{l}\text { I. Para-aminobenzoate synthase, amidotransferase component (EC 2.6.1.85) @ Anthranilate synthase, } \\
\text { amidotransferase component (EC 4.1.3.27) }\end{array}$ \\
\hline & FIG00000252 & m. 5-formyltetrahydrofolate cyclo-ligase (EC 6.3.3.2) \\
\hline
\end{tabular}

superoxide dismutase ( 2 genes) and a catalase gene.

B106 also possessed Toxin-antitoxin (TA) protein such as MazEF and HigA (Table 6) that are involved in stress responses. The role of TAs encoded by bacterial chromosomes is not fully understood [54], however MazF protein has been recently categorized as an endoribonuclease or as a type of RNA interference enzyme [55] that induces apoptosis in response to starvation, precisely due to amino acid deficiency [56]. TAs have been suggested to function as stress-responsive elements and it's programmed cell death aids in survival of remaining bacterial population during stress with the nutrients released by the dead cells, a kind of "nutritional-altruism" [57]. 
Table 6. Putative Toxin-antitoxin system involved stress related genes detected in B106.

\begin{tabular}{llc}
\hline \multicolumn{1}{c}{ Function } & \multicolumn{1}{c}{ Evidence codes } & Figfam \\
\hline Programmed cell death antitoxin YdcD & YdcE-YdcD toxin-antitoxin (programmed cell death) systems. & FIG00008524 \\
& MazEF toxin-antitoxin (programmed cell death) system & \\
Programmed cell death toxin YdcE & YdcE-YdcD toxin-antitoxin (programmed cell death) systems. & FIG00009628 \\
& MazEF toxin-antitoxin (programmed cell death) system & \\
Lmo0066 homolog within ESAT-6 gene cluster, & ESAT 6 proteins secretion system in Firmicutes & FIG00022837 \\
similarity to ADP-ribosylating toxins & & \\
HigA protein (antitoxin to HigB) & Toxin-antitoxin replicon stabilization systems & FIG00010902 \\
\hline
\end{tabular}

The cells are protected from high osmolarity environments by presence of Glycine betaine. High osmolarity induces de novo synthesis OpuD protein and stimulates the established $\mathrm{OpuD}$ proteins to uptake glycine betaine at maximal levels [58]. In B106, 4 copies of OpuD genes and 2 copies of OpuAB, OPU AC, and 1 copy each of OpuAA, permease protein proW and proV were detected, indicating the high osmotolerant ability of the strain B106.

Antibiotic resistant proteins in B106: Probiotic strains can ideally carry few antibiotic resistance genes, so that probiotics can be taken along with antibiotic treatment for restoring the normal gut microflora and to prevent gastrointestinal distress and antibiotic-associated diarrhea [59]. B106 genome was mined for the presence of genes encoding antibiotic resistance and related efflux pumps using Resistance Gene Identifier (RGI) option in CARD web server. The CARD includes a curated collection of antimicrobial resistance gene and mutation sequences, and the software Resistance Gene Identifier (RGI) for their detection in genome or protein sequences [60].

The heatmap (Fig. 4) represents the relatedness between the genes and their functional categories identified in the genome. Perfect indicates $100 \%$ similarity matching with the functional category in the database, whereas Strict indicates they are less than $100 \%$ similar, however, they have fall in the same functional category identified.

ANT(4')-Ia an aminoglycoside resistance and $c l b c$ and cat - Phenicol resistance, Erm(34) (Macrolide resistance), beta-lactam resistance - blaI, and determinants for linezolid resistance, macrolide resistance, streptogramin resistance and lincosamide resistance were detected in B106 genome.
RAST annotation of B106 genome also revealed the presence of genes encoding resistance to beta-lactamas, Chloramphenicol, vancomycin, tetracycline, fluoroquinolone, Kanamycin and fosfomycin. Also, large numbers of MFS and other efflux pumps such as MATE (multidrug and toxic efflux) and RND (resistance-nodulation-division) were also detected (Table 7).

AntiSMASH server, revealed the presence of biosynthetic gene clusters for Lantipeptide and Ecotine in B106. Lantipepetide is an antibacterial peptide whereas Ecotine is a serine protease inhibitor, protecting the bacteria against antibacterial activity of neutrophil elastase [61].

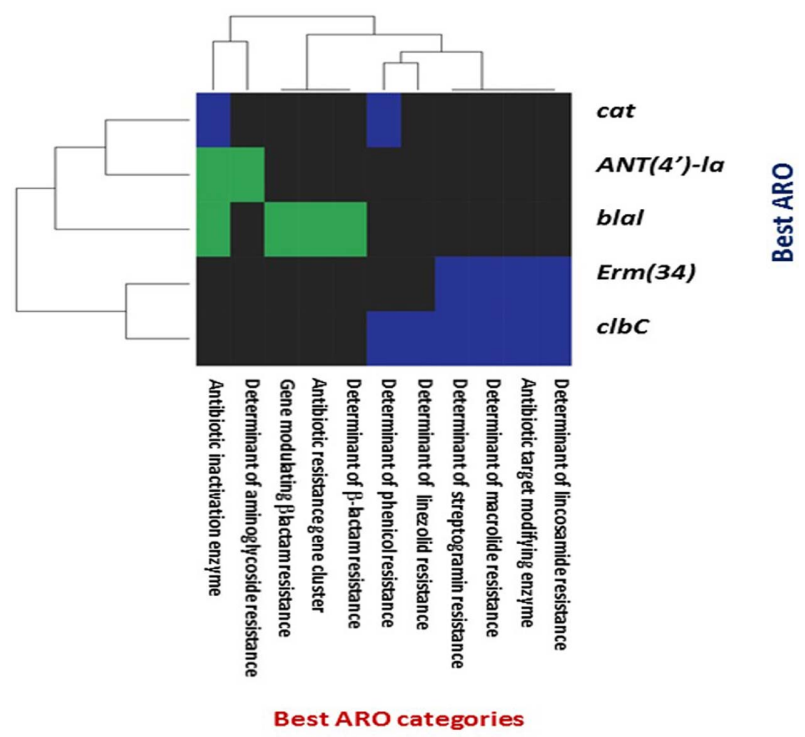

Fig. 4. Heat map of genes encoding antibiotic resistance in B106 as putatively identified by Resistance Gene Identifier. Perfect ( $\square$ ) and strict ( $\square$ ) matches of putative genes are illustrated. Key to abbreviation- cat: chloramphenicol acetyltransferase; ANT (4') la: adenyltransferases; blal: $\beta$-Lactamases; Erm (34): Efflux rRNA methylase; clbC: colibactin; indicates N/A. 
Table 7. Putative antibiotic resistance genes detected in B106.

\begin{tabular}{lc}
\hline \multicolumn{1}{c}{ Putative genes } & Figfam \\
\hline Bicyclomycin resistance protein & FIG00452786 \\
Chloramphenicol resistance protein & - \\
Drug resistance transporter EmrB/QacA subfamily & FIG00628872 \\
drug resistance transporter, EmrB/QacA family & FIG01265370 \\
Fosfomycin resistance protein FosB & FIG00014222 \\
$\begin{array}{l}\text { Glyoxalase/bleomycin resistance protein/ } \\
\text { dioxygenase }\end{array}$ & - \\
$\begin{array}{l}\text { Hypothetical protein DUF901, similar to C-terminal } \\
\text { domain of ribosome protection-type Tc-resis- }\end{array}$ & FIG00001549 \\
tance proteins & \\
Kanamycin kinase (Aminoglycoside phospho- & FIG01867116 \\
transferase, gentamicin resistance protein) & \\
(EC 2.7.1.95) & \\
$\begin{array}{l}\text { Multidrug resistance protein B } \\
\text { Multiple antibiotic resistance protein marC }\end{array}$ & \\
oxetanocin A resistance protein & FIG01356213 \\
Ribosome protection-type tetracycline resis- & FIG00007998 \\
tance related proteins & \\
RND multidrug efflux transporter; Acriflavin & FIG00132942 \\
resistance protein & \\
Teicoplanin resistance protein vanZ & \\
Vancomycin B-type resistance protein VanW & FIG00003433 \\
\hline
\end{tabular}

Though, several antibiotic resistance proteins were detected, none of them were found to be present on transposable elements, eliminating the possibility of horizontal gene transfer of resistance genes from B106 to any other pathogens. Absence of plasmids in B106, further confirmed that the strain does not harbor any transmissible antibiotic resistance traits, making it suitable for use as a probiotic.

Thus, genome analysis of B106 revealed the presence of candidate genes responsible for gut survival and adaptation, adhesion, health promotion and disease prevention. B106 may be regarded as a nutrient factory, as vitamin synthesizing genes and CAZymes were detected in B106 genome. The ability of B106 to produce bacteriocins and antibiotics confer it with a selective advantage to competitively exclude pathogenic microorganisms from the gut and prevent gastrointestinal disorders and antibiotic-induced diarrhea. Presence of sporulation genes provides an added advantage to B106, making it a resilient and stable probiotic. Our study highlighted the probiotic potential of B106 and encourages further studies to get insights into the immunomodulatory properties of this strain.

\section{Acknowledgment}

Financial assistance from $\mathrm{Hi}$ Tech BioSciences India Ltd. is gratefully acknowledged.

CSIR Senior Research Fellowship to Neelam Kapse is gratefully acknowledged.

\section{Conflict of Interest}

The authors have no financial conflicts of interest to declare.

\section{References}

1. Fuller R. 1989. Probiotics in man and animals. J. Appl. Bacteriol. 66: 365-378.

2. Mazza P. 1994. The use of Bacillus subtilis as an antidiarrhoeal microorganism. Boll. Chim. Farm. 133: 3-18.

3. Hoa TT, Duc LH, Isticato R, Baccigalupi L, Ricca E, Van PH, et al. 2001. Fate and dissemination of Bacillus subtilis spores in a murine model. Appl. Environ. Microbiol. 67: 3819-3823.

4. Bader J, Albin A, Stahl U. 2012. Spore-forming bacteria and their utilisation as probiotics. Benef Microbes. 3: 67-75.

5. Lefevre M, Racedo SM, Ripert G, Housez B, Cazaubiel M, Maudet C, et al. 2015. Probiotic strain Bacillus subtilis CU1 stimulates immune system of elderly during common infectious disease period: a randomized, double-blind placebo-controlled study. Immun. Ageing 12: 24.

6. Shobharani P, Padmaja RJ, Halami PM. 2015. Diversity in the antibacterial potential of probiotic cultures Bacillus licheniformis MCC2514 and Bacillus licheniformis MCC2512. Res. Microbiol. 166: 546-554.

7. Ripert G, Racedo SM, Elie AM, Jacquot C, Bressollier P, Urdaci MC. 2016. Secreted compounds of the probiotic bacillus clausii strain $\mathrm{O} / \mathrm{C}$ inhibit the cytotoxic effects induced by Clostridium difficile and Bacillus cereus toxins. Antimicrob. Agents Chemother. 60: 3445-3454.

8. Pham M, Lemberg DA, Day AS. 2008. Probiotics: sorting the evidence from the myths. Med. J. Aust. 188: 304-308.

9. Hong HA, Duc le LH, Cutting SM. 2005. The use of bacterial spore formers as probiotics. FEMS Microbiol. Rev. 29: 813-835.

10. Lakshmi SG, Jayanthi N, Saravanan M, Ratna MS. 2017. Safety assesment of Bacillus clausii UBBC07, a spore forming probiotic. Toxicol. Rep. 4: 62-71.

11. Urdaci MC, Bressollier P, Pinchuk I. 2004. Bacillus clausii probiotic strains: antimicrobial and immunomodulatory activities. J. Clinic Gastroenterol. 38: S86-S90.

12. Upadrasta A, Pitta S, Madempudi RS. 2016. Draft genome sequence of Bacillus clausii UBBC07, a spore-forming probiotic 
strain. Genome Announc. 4: e00235-16.

13. Bankevich A, Nurk S, Antipov D, Gurevich AA, Dvorkin M, Kulikov AS, et al. 2012. SPAdes: a new genome assembly algorithm and its applications to single-cell sequencing. J. Comput. Biol. 19:455477.

14. Aziz RK, Bartels D, Best AA, DeJongh M, Disz T, Edwards RA, et al. 2008. The RAST Server: rapid annotations using subsystems technology. BMC Genomics. 9: 75.

15. Auch AF, Jan M, Klenk HP, Göker M. 2010. Digital DNA-DNA hybridization for microbial species delineation by means of genome-to-genome sequence comparison. Stand Genomic. Sci. 28: $117-134$

16. Alikhan NF, Petty NK, Zakour NLB, Beatson SA. 2011. BLAST Ring Image Generator (BRIG): simple prokaryote genome comparisons. BMC Genomics. 12: 402.

17. Weber T, Blin K, Duddela S, Krug D, Kim HU, Bruccoleri R, et al. 2015. antiSMASH 3.0-a comprehensive resource for the genome mining of biosynthetic gene clusters. Nucleic Acids Res. 43: W237-W243.

18. van Heel AJ, de Jong A, Montalban-Lopez M, Kok J, Kuipers OP. 2013. BAGEL3: automated identification of genes encoding bacteriocins and (non-) bactericidal posttranslationally modified peptides. Nucleic Acids Res. 41: W448-W453.

19. Jia B, Raphenya AR, Alcock B, Waglechner N, Guo P, Tsang KK, et al. 2016. CARD 2017: expansion and model-centric curation of the comprehensive antibiotic resistance database. Nucleic Acids Res. 45: D566-D573.

20. Holzapfel WH, Haberer P, Geisen R, Björkroth J, Schillinger U. 2001. Taxonomy and important features of probiotic microorganisms in food and nutrition. Am. J. Clin. Nutr. 73: 365s-373s.

21. Han XY. 2006. Bacterial identification based on $16 \mathrm{~S}$ ribosomal RNA gene sequence analysis. In: Advanced techniques in diagnostic microbiology, Springer, Boston, MA, pp. 323-332.

22. Sleator RD, Watson D, Hill C, Gahan CG. 2009. The interaction between Listeria monocytogenes and the host gastrointestinal tract. Microbiology 155: 2463-2475.

23. Toledo-Arana A, Dussurget O, Nikitas G, Sesto N, Guet-Revillet H, Balestrino $D$, et al. 2009. The Listeria transcriptional landscape from saprophytism to virulence. Nature 459: 950-956.

24. Ryan S, Hill C, Gahan CG. 2008. Acid stress responses in Listeria monocytogenes. Adv. Appl. Microbiol. 65: 67-91.

25. Cotter PD, Hill C. 2003. Surviving the acid test: responses of gram-positive bacteria to low pH. Microbiol. Mol. Biol. Rev. 67: 429-453.

26. Hain T, Hossain H, Chatterjee SS, Machata S, Volk U, Wagner S, et al. 2008. Temporal transcriptomic analysis of the Listeria monocytogenes EGD-e $\sigma$ B regulon. BMC Microbiol. 8: 20.

27. Lehri B, Seddon AM, Karlyshev AV. 2017. Potential probiotic-associated traits revealed from completed high quality genome sequence of Lactobacillus fermentum 3872. Stand Genomic Sci. 12: 19.

28. Khatri I, Sharma S, Ramya TNC, Subramanian S. 2016. Complete genomes of Bacillus coagulans S-lac and Bacillus subtilis TO-A
JPC, two phylogenetically distinct probiotics. PLoS One. 11: e0156745.

29. Begley M, Gahan CG, Hill C. 2005. The interaction between bacteria and bile. FEMS Microbiol. Re. 29: 625-651.

30. McAuliffe O, Cano RJ, Klaenhammer TR. 2005. Genetic analysis of two bile salt hydrolase activities in Lactobacillus acidophilus NCFM. Appl. Environ. Microbiol. 71: 4925-4929.

31. Denou E, Pridmore RD, Berger B, Panoff JM, Arigoni F, Brüssow H. 2008. Identification of genes associated with the long-gut-persistence phenotype of the probiotic Lactobacillus johnsonii strain NCC533 using a combination of genomics and transcriptome analysis. J. Bacteriol. 190: 3161-3168.

32. Lim SM. 2014. Antimutagenicity activity of the putative probiotic strain Lactobacillus paracasei subsp. tolerans JG22 isolated from pepper leaves Jangajji. Food Sci. Biotechnol. 23: 141-150.

33. Granato D, Bergonzelli GE, Pridmore RD, Marvin L, Rouvet $M$, Corthésy-Theulaz IE. 2004. Cell surface-associated elongation factor Tu mediates the attachment of Lactobacillus johnsonii NCC533 (La1) to human intestinal cells and mucins. Infect. Immun. 72: 2160-2169.

34. Howarth GS, Wang H. 2013. Role of endogenous microbiota, probiotics and their biological products in human health. Nutrients 5: 58-81.

35. Ruas-Madiedo $P$, Gueimonde $M$, Margolles $A$, de los REYESGAVILÁN CG, Salminen S. 2006. Exopolysaccharides produced by probiotic strains modify the adhesion of probiotics and enteropathogens to human intestinal mucus. J. Food Prot. 69: 20112015.

36. Kodali VP, Sen R. 2008. Antioxidant and free radical scavenging activities of an exopolysaccharide from a probiotic bacterium. Biotechnol. J. 3: 245-251.

37. Haiko J, Westerlund-Wikström B. 2013. The role of the bacterial flagellum in adhesion and virulence. Biology 2: 1242-1267.

38. Cotter PD, Ross RP, Hill C. 2013. Bacteriocins-a viable alternative to antibiotics?. Nat. Rev. Microbiol. 11: 95-105.

39. Lv LX, Li YD, Hu XJ, Shi HY, Li LJ. 2014. Whole-genome sequence assembly of Pediococcus pentosaceus LI05 (CGMCC 7049) from the human gastrointestinal tract and comparative analysis with representative sequences from three food-borne strains. Gut. Pathog. 6: 36.

40. Cotter PD, Hill C, Ross RP. 2005. Food microbiology: bacteriocins: developing innate immunity for food. Nat. Rev. Microbiol. 3: 777 788.

41. González-Martínez BE, Gómez-Treviño, Jiménez-Salas Z. 2003. Bacteriocinas de probióticos. Rev Cub Salud Publica. 4.

42. Hertzberger R, Arents J, Dekker HL, Pridmore RD, Gysler C, Kleerebezem $\mathrm{M}$, et al. 2014. $\mathrm{H}_{2} \mathrm{O}_{2}$ production in species of the Lactobacillus acidophilus group: a central role for a novel NADHdependent flavin reductase. Appl. Environ. Microbiol. 80: 22292239.

43. Galperin MY, Mekhedov SL, Puigbo P, Smirnov S, Wolf YI, Rigden DJ. 2012. Genomic determinants of sporulation in Bacilli and Clostridia: towards the minimal set of sporulation-specific genes. 
Environ. Microbiol. 14: 2870-2890.

44. Roberfroid MB. 2002. Prebiotics and probiotics: are they functional foods?. Am. J. Clin. Nutr. 71: 1682S-1687S.

45. Campbell JM, Fahey Jr GC, Wolf BW. 1997. Selected indigestible oligosaccharides affect large bowel mass, cecal and fecal shortchain fatty acids, pH and microflora in rats. J. Nutr. 127: 130-136.

46. Gibson GR, McCartney AL, Rastall RA. 2005. Prebiotics and resistance to gastrointestinal infections. Br. J. Nutr. 93: S31-S34.

47. Grootaert C, Van den Abbeele P, Marzorati M, Broekaert WF, Courtin CM, Delcour JA, et al. 2009. Comparison of prebiotic effects of arabinoxylan oligosaccharides and inulin in a simulator of the human intestinal microbial ecosystem. FEMS Microbiol. Ecol. 69: 231-242.

48. Gueimonde M, Noriega L, Margolles A, Clara G. 2007. Induction of a-l-arabinofuranosidase activity by monomeric carbohydrates in Bifidobacterium longum and ubiquity of encoding genes. Arch. Microbiol. 187: 145-153.

49. Di Pierro F, Bertuccioli A, Marini E, Ivaldi L. 2015. A pilot trial on subjects with lactose and/or oligosaccharides intolerance treated with a fixed mixture of pure and enteric-coated $\alpha$-and $\beta$-galactosidase. Clin. Exp. Gastroenterol. 8: 95-100.

50. Parche S, Amon J, Jankovic I, Rezzonico E, Beleut M, Barutçu H, et al. 2007. Sugar transport systems of Bifidobacterium longum NCC2705. J. Mol. Microbiol. Biotechnol. 12: 9-19.

51. Preidis GA, Hill C, Guerrant RL, Ramakrishna BS, Tannock GW, Versalovic J. 2011. Probiotics, enteric and diarrheal diseases, and global health. Gastroenterology 140: 8-14.

52. Saulnier DM, Santos F, Roos S, Mistretta TA, Spinler JK, Molenaar $D$, et al. 2011. Exploring metabolic pathway reconstruction and genome-wide expression profiling in Lactobacillus reuteri to define functional probiotic features. PLoS One 6: e18783.

53. Mills S, Stanton C, Fitzgerald GF, Ross R. 2011, December. Enhancing the stress responses of probiotics for a lifestyle from gut to product and back again. Microb. Cell Fact. 10: S19.

54. Van Melderen L. 2010. Toxin-antitoxin systems: why so many, what for?. Curr. Opin. Microbiol. 13: 781-785.

55. Yan X, Gurtler JB, Fratamico PM, Hu J, Juneja VK. 2012. Phylogenetic identification of bacterial MazF toxin protein motifs among probiotic strains and foodborne pathogens and potential implications of engineered probiotic intervention in food. Cell Biosci. 2: 39.

56. Aizenman E, Engelberg-Kulka H, Glaser G. 1996. An Escherichia coli chromosomal" addiction module" regulated by guanosine [corrected] 3', 5'-bispyrophosphate: a model for programmed bacterial cell death. Proc. Natl. Acad. Sci. USA 93: 6059-6063.

57. Ramisetty BCM, Natarajan B, Santhosh RS. 2015. mazEF-mediated programmed cell death in bacteria: "what is this?". Crit. Rev. Microbiol. 41: 89-100.

58. Kappes RM, Kempf B, Bremer E. 1996. Three transport systems for the osmoprotectant glycine betaine operate in Bacillus subtilis: characterization of OpuD. J. Bacteriol. 178: 5071-5079.

59. Gueimonde M, Sánchez B, de los Reyes-Gavilán CG, Margolles A. 2013. Antibiotic resistance in probiotic bacteria. Front Microbiol. 4: 202.

60. McArthur AG, Waglechner N, Nizam F, Yan A, Azad MA, Baylay AJ, et al. 2013. The comprehensive antibiotic resistance database. Antimicrob. Agents Chemother. 57: 3348-3357.

61. Eggers CT, Murray IA, Delmar VA, Day AG, Craik CS. 2004. The periplasmic serine protease inhibitor ecotin protects bacteria against neutrophil elastase. Biochem. J. 379: 107-118. 